Martyna Gębska-Tołoczko ${ }^{1}$, Jakub J. Kałużny ${ }^{1,3}$, Agata Żaroń2, Beata Danek ${ }^{3}$, Joanna Pulkowska-Ulfig², Oliwia Beck², Milena Wojciechowska², Bartłomiej J. Kałużny ${ }^{3,4}$

Department of Biology of the Visual System, Collegium Medicum, Nicolaus Copernicus University, Bydgoszcz, Poland

2Department of Public Health, Collegium Medicum, Nicolaus Copernicus University, Bydgoszcz, Poland

${ }^{3}$ Oftalmika Eye Hospital, Bydgoszcz, Poland

${ }^{4}$ Department of Optometry, Collegium Medicum, Nicolaus Copernicus University, Bydgoszcz, Poland

\title{
Cognitive functions in patients after cataract phacoemulsification and implantation of multifocal and monofocal intraocular lenses
}

Presented at the XXXIV Congress of the European Society of Cataract \& Refractive Surgeons, Copenhagen, Denmark, September 2016

\section{Corresponding author:}

Jakub J. Kałużny, ul. Sandomierska 16, $85-830$ Bydgoszcz, Poland Tel: +58 525855408 e-mail: jakubkaluzny@cm.umk.pl
Medical Research Journal 2018; Volume 3. Number 2, 70-75 10.5603/MRJ.2018.0012 Copyright $\odot 2018$ Via Medica ISSN 2451-2591

\begin{abstract}
Introduction: The study has to determine whether implantation of diffractive multifocal lenses during cataract phacoemulsification causes distortion of the cognitive aspects of processing perceptual information.

Material and methods: 25 patients who underwent cataract phacoemulsification with the implantation of diffractive multifocal lens Acrysof Restor SN6AD1 in both eyes (multifocal group) and 26 patients with the implantation of monofocal intraocular lens Acrysof IQ SN60WF in both eyes (monofocal group) were enrolled. All patients underwent ophthalmic examination and cognitive function- Trial Making Test (Part A and Part B) and Stroop Test (Stroop Test 1 and Stroop Test 2) tests at least 6 months after cataract surgery of the other eye. Results: Mean patient age and visual acuity with correction for near and far vision did not differ between groups. Average Trail Making scores were $32.80 \pm 11.86 \mathrm{~s}$ and $84.08 \pm 33.26 \mathrm{~s}$ for Parts $A$ and $B$, respectively, in the multifocal group, while scores in the monofocal group were $36.61 \pm 13.63 \mathrm{~s}$ and $93.34 \pm 40.49 \mathrm{~s}$ in Parts $A$ and B. Stroop Test scores were $79.09 \pm 17.69 \mathrm{~s}$ and $133.64 \pm 17.60 \mathrm{~s}$ for Stroop Test 1 and Stroop Test 2, respectively, in the multifocal group; in the monofocal group, scores were $82.04 \pm 17.51 \mathrm{~s}$ and $152.88 \pm 65.72 \mathrm{~s}$, respectively. The groups did not differ for either test.

Conclusions: Differences between cognitive function tests results between patients with multifocal and monofocal intraocular lenses were not statistically significant suggesting the lack of influence of the type of lens on visual perception at least in good light conditions. Further clinical trials using more sophisticate tests in different light conditions are needed.

Key words: Multifocal intraocular lenses, cataract phacoemulsification, cognitive function, visual perception
\end{abstract}

\section{Introduction}

Multifocal lenses are used more and more frequently to treat cataract and presbyopia. They allow patients to achieve a visual acuity for far vision similar to monofocal lenses and to become independent of eyeglasses for near vision [1-6]. Multifocal diffractive lenses work by creating separate focal points for objects that are near and far away. In the vision process, the brain chooses a focus with a better image quality, which depends on the position of the object being observed. At the same time, however, a sharp image overlaps another, blurred image, resulting in decreased sensitivity to contrast and in retinal sensitivity to light in the perimetry [7]. Light diffusion associated with the construction of multifocal lenses is responsible for the appearance of halo and glare, and patients may experience blurry vision or alterations in vision acuity [8-12]. These complaints decrease for most patients with time from lens implantation, which is associated with the gradual adaptation of the visual cortex to new visual conditions called 'neuroadaptation' [7, 13-14]. Issues associated with neuroadaptation disorders are among the most common causes of multifocal lens explantation [6]. 
Compared with monofocal lenses, the reduced image quality associated with intraocular multifocal lenses and the overlapping of images may affect cognitive functions. Cognitive function is defined by a set of dynamic processes occurring in the mind, through which information from the environment is received, processed, and reintroduced into the environment in the form of a reaction. Here we used a neuropsychological perspective that identifies cognitive functions as one of the dimensions of human functioning [15]. In this view, they are divided into elementary and complex cognitive processes through which the mind receives and interprets stored information and communicates it externally. Elementary cognitive processes include perception, memory, attention, and cognitive control. Complex ones comprise thinking and understanding, problem solving, judgment, language, and speech.

Visual perception is one of the information-processing activities that are an example of elementary cognitive processes. The process of visual perception is the process of object representation based on the information received from the sensory organ and the information contained in memory. The perception - that is, the image of an object, taking into account all its available features - is the effect of visual cortex activity, which receives information from the various senses and merges them together [16]. Cognitive functions such as attention, memory, and cognitive control, which are essential for the active interpretation of visual stimuli, are involved in the individual stages of cognitive functioning.

The aim of this study was to determine whether implantation of diffractive multifocal lenses during cataract phacoemulsification causes the distortion of cognitive aspects of processing perceptual information, which include attention, working memory, and cognitive control.

\section{Material and methods}

This observational case control study was conducted at the Department of Biology of the Visual System, Collegium Medicum, Nicolaus Copernicus University in Bydgoszcz, Poland between June 2015 and March 2016. This study followed the tenets of the Declaration of Helsinki. Informed consent was obtained from the subjects after explaining the nature and possible consequences of the study. The research was approved by the Ethics Committee of the institution where all authors were affiliated.

Patients who underwent cataract phacoemulsification in both eyes were included in the study and divided into two groups. The first group had a diffractive multifocal lens (AcrySof Restor SN6AD1) implanted in both eyes, and the second group had a monofocal lens (AcrySof IQ SN60WF) implanted in both eyes.
Inclusion criteria covered postoperative visual acuity with correction above 0.8 and no other ocular diseases including retinal disease, glaucoma, optic nerve neuropathy, corneal disease, preoperative refractive error above $\pm 3.0 \mathrm{Dsph}$, astigmatism above $\pm 1.0 \mathrm{Dcyl}$, and amblyopia. All cataract phacoemulsification operations were performed by one surgeon in an uncomplicated manner with an incision in a transparent cornea. The interval between the operation of one eye and the other eye did not exceed 12 months. Patients with mental disorders like depressive and anxiety disorders, as well as mild cognitive impairment and neurocognitive disorders correlated with age like dementia were excluded. Verification of these based on an anamnesis towards neurological disorders and basic neurological exam took place at the same day before the cognitive function tests were performed.

All patients underwent ophthalmic examination and cognitive function tests at least 6 months after the second eye cataract surgery. The ophthalmic examination included visual acuity assessment on ETDRS charts for far vision with and without correction as well as the visual acuity assessment for near vision with and without correction. Intraocular pressure, the anterior segment of the eye, and retinal state were measured or examined to exclude any pathology.

For evaluating cognitive functions, we used the Trail Making Test (TMT; Part A and Part B) and Stroop Test (Stroop Test 1 and Stroop Test 2). The TMT was used to measure visual field searching efficiency and psychomotor speed (Part A). It also measures the processes of attention, working memory, and cognitive flexibility in visual-spatial material (Part B) [17-18]. The test consists of combining with a solid line 25 randomly arranged numbers in ascending order on an A4 paper size (Part $A$ ) and 25 randomly arranged numbers in ascending order and according to the rules of the alphabet (Part B). The performance time of each part measured in seconds was considered when evaluating the results. The time difference of both Parts $(B-A)$ was also calculated, which allowed for evaluation of cognitive memory and cognitive flexibility, also called 'switching' [8, 19].

The Stroop Test is one of the most popular methods of assessing cognitive control with reference to interference [18]. The test material consists of two tasks. The first (Stroop Test 1 ) is to call out the print color as fast as possible, and the second (Stroop Test 2) is an interference task that requires noting a conflict between the meaning of the word and the color in which it is written. The purpose of the test is to evaluate the effectiveness of material selection through cognitive suppression or interference, which in this task consists of suppressing the dominant reaction of the automatic reading process and replacing it with a new 
Table 1. Demographic data

\begin{tabular}{lccc}
\hline & $\begin{array}{c}\text { Multifocal IOL } \\
(\mathbf{n = 2 5 )}\end{array}$ & $\begin{array}{c}\text { Monofocal IOL } \\
(\mathbf{n = 2 6 )}\end{array}$ & $\mathbf{p}$ \\
\hline Sex (women:men) & $12: 13$ & $14: 12$ & \\
Age (y) & $64.52 \pm 4.80$ & $64.58 \pm 4.96$ & 0.967 \\
AL right eye (mm) & $23.57 \pm 1.32$ & $23.64 \pm 1.44$ & 0.872 \\
AL left eye (mm) & $23.57 \pm 1.31$ & $23.71 \pm 1.50$ & 0.712 \\
Mean IOL power right eye (D) & $21.86 \pm 4.23$ & $21.71 \pm 3.54$ & 0.947 \\
Mean IOL power left eye (D) & $22.05 \pm 4.38$ & $21.67 \pm 3.72$ & 0.748 \\
Time elapsed after surgery (d) - right eye & $608.28 \pm 44.49$ & $473.77 \pm 56.86$ & 0.008 \\
Time elapsed after surgery (d) - left eye & $580.48 \pm 49.59$ & $502.54 \pm 77.83$ & 0.015 \\
\hline
\end{tabular}

$\mathrm{AL}$ - axial length

$\mathrm{IOL}$ - intraocular lens

Table 2. Postoperative refractive error

\begin{tabular}{|c|c|c|c|}
\hline & $\begin{array}{l}\text { Multifocal IOL } \\
\quad(n=25)\end{array}$ & $\begin{array}{c}\text { Monofocal IOL } \\
(n=26)\end{array}$ & $\mathbf{p}$ \\
\hline \multicolumn{4}{|c|}{$\begin{array}{l}\text { Mean refractive error for far vision } \\
\text { (spherical equivalent, Dsph) }\end{array}$} \\
\hline — right eye & $0.085 \pm 0.33$ & $-0.88 \pm 1.33$ & 0.001 \\
\hline - left eye & $0.035 \pm 0.39$ & $-0.71 \pm 1.24$ & 0.015 \\
\hline \multicolumn{4}{|c|}{$\begin{array}{l}\text { Mean refractive error for near vision } \\
\text { (spherical equivalent, Dsph) }\end{array}$} \\
\hline — right eye & $0.45 \pm 0.80$ & $1.43 \pm 1.20$ & 0.006 \\
\hline — left eye & $0.50 \pm 0.83$ & $1.64 \pm 1.10$ & 0.001 \\
\hline
\end{tabular}

reaction, the naming of the font color. The distractor that hinders the suppression is the color significance. When evaluating the results, the time taken for each sample was analyzed, and the time difference of the interference task and the control task was also evaluated as an indicator of interference.

TMT Part A and Part B, Stroop Test 1, and Stroop Test 2 were performed in all patients in the same room and with the same lighting conditions in the presence of a qualified psychologist. High-intensity artificial lighting for easy reading was used. Patients who used reading glasses daily did the tests with correction for near vision, and all others did them without correction. The test cards were at a standard distance of $40 \mathrm{~cm}$.

\section{Statistical analysis}

The Shapiro-Wilk test was used to resolve the normality distribution of the data. Parametric comparisons were made using a $t$-test and non-parametric comparisons were made using the Mann-Whitney $U$ test. Spearman's correlation coefficients between cognitive function tests and visual parameters were also calculated. Probability values of $p<0.05$ were considered statistically significant.

\section{Results}

A total of 25 patients who underwent cataract phacoemulsification with the implantation of diffractive multifocal lens in both eyes (multifocal group) and 26 patients with the implantation of monofocal intraocular lens in both eyes (monofocal group) were enrolled. The demographic data of patients are presented in Tab. 1. Differences between mean age and eye length were not statistically significant. For patients in the multifocal group, the interval between surgery and control was greater than in the monofocal group.

Tables 2 and 3 show the results of postoperative visual acuity and correction in the study groups. In the monofocal group, the mean postoperative refractive error was shifted to myopia and was $-0.88 \pm 1.33 \mathrm{Dsph}$ in the right eye and $-0.71 \pm 1.24 \mathrm{Dsph}$ in the left eye. In this group, the patients required significantly greater correction for near vision in comparison with the eyes implanted with multifocal lenses. Visual acuity with correction for far vision and for near vision did not differ significantly between the groups.

Twelve patients (48\%) from the multifocal group reported total independence from using eyeglasses. Only two people in this group used eyeglasses for near vision on a constant basis, and eleven patients reported doing 
Table 3. Postoperative visual acuity

$\begin{array}{ccc}\begin{array}{c}\text { Multifocal IOL } \\ (n=25)\end{array} & \begin{array}{c}\text { Monofocal IOL } \\ (n=26)\end{array} & p\end{array}$

Mean visual acuity for far vision with correction,

logMar

— right eye

$0.0128 \pm 0.12$

$-0.0108 \pm 0.18$

- left eye

$0.0128 \pm 0.11$

$-0.0038 \pm 0.17$

0.130

Mean visual acuity for near vision with correction,

logMar

— right eye

$0.152 \pm 0.09$

$0.111 \pm 0.13$

0.107

- left eye

$0.128 \pm 0.08$

$0.104 \pm 0.13$

0.187

Table 4. Mean results of cognitive tests

\begin{tabular}{lccc}
\hline & $\begin{array}{c}\text { Multifocal IOL } \\
(\mathbf{n = 2 5 )}\end{array}$ & $\begin{array}{c}\text { Monofocal IOL } \\
(\mathbf{n}=\mathbf{2 6})\end{array}$ & $\mathbf{p}$ \\
Trail Making Test, Part A (s) & $32.80 \pm 11.86$ & $36.61 \pm 13.63$ & 0.292 \\
Trail Making Test, Part B (s) & $84.08 \pm 33.26$ & $93.34 \pm 40.49$ & 0.377 \\
Trail Making Test, Part B - A (s) & $51.28 \pm 30.86$ & $56.73 \pm 36.11$ & 0.566 \\
Stroop Test 1 (s) & $79.09 \pm 17.69$ & $82.04 \pm 17.51$ & 0.631 \\
Stroop Test 2 (s) & $133.64 \pm 17.60$ & $152.88 \pm 65.72$ & 0.386 \\
Stroop Test 2 - 1 (s) & $64.04 \pm 37.57$ & $74.00 \pm 71.84$ & 0.685 \\
\hline
\end{tabular}

so only occasionally (44\%). In the monofocal group, all patients used eyeglasses for near vision, with constant correction required in 18 patients $(69.2 \%)$ and occasional correction in 8 (30.8\%). Sporadic occurrence of glare or halo occurred in $16(64 \%)$ patients in the multifocal group and 4 (16\%) in the monofocal group.

The TMT results are shown in Tab. 4. For both Part $A$ and $P$ art $B$ and the differences between $B$ and $A$ $(B-A)$, the mean test times were lower for the multifocal group, yet these differences were not statistically significant. A similar pattern occurred with the Stroop Test (Tab. 4), where the observed differences between the groups were not statistically significant. TMT and Stroop Test results did not correlate significantly with postoperative refractive error, visual acuity with far and near vision, eye length, power of the implanted intraocular lens, or the time distance between the operation and control in both groups. Scores also showed no significant correlation with patient age.

\section{Discussion}

The study compared two groups of patients of similar age, eye length, and power of implanted intraocular lenses. One group of patients had diffractive multifocal lenses implanted for both eyes during cataract phacoemulsification, and the second group received monofocal lenses. The results obtained after the oper- ation with reference to visual acuity with correction for far and near vision did not differ significantly between groups. The use of multifocal lenses allowed $48 \%$ of those patients to be completely independent of eyeglasses whereas no patients in the monofocal group were eyeglasses free. TMT and Stroop Test scores did not differ between groups with reference to cognitive functions in terms of visual field efficiency and psychomotor speed, as well as for attention, memory, control, and cognitive flexibility.

All available publications confirm the benefits of using multifocal lenses, which improve near vision without correction in comparison with monocular lenses [1-5, $11-12$ ]. In $7 \%$ to $69 \%$, total independence from eyeglasses is achieved, and almost all have reduced need for eyeglasses [2]. Unfortunately, such lenses have associated costs, including decreased contrast sensitivity, especially under mesotopic lighting conditions, as well as glare and halo [1-5]. In the study material, this phenomenon occurred in $64 \%$ of the patients. Negative phenomena accompanying diffractive multifocal lenses are associated with image formation and loss of light energy dissipating on the lens surface [2-5]. Because of the concentric rings located on the surface of the diffractive lens, the light rays are diffracted so that two foci are formed, one for far and the other for near vision. As a result, the non-target blurred image always overshadows the targeted sharp image, which hinders vision and causes ailments that, in extreme cases, even necessitate lens explantation [6]. 
The image overlap of observed retinal objects in patients with implanted diffractive intraocular lenses distorts the processes of image analysis within the visual cortex. Studies using functional magnetic resonance imaging have demonstrated that, unlike in patients with monofocal lenses, cortical centers in patients with implanted multifocal lenses are activated under poor lighting conditions; among other things, these regions are responsible for task planning and problem solving $[18,20]$. Thanks to the process of neuroadaptation, in the majority of patients, these problems decrease with the time that has elapsed since surgery [13-14, 19, 21]. This process lasts for at least 6 months after the surgery, although it slows or stops completely in some patients and in exceptional cases leads to lens removal. The neuroadaptation process can be significantly accelerated with special training programs; however, some disorders persist despite a long adaptation time, including reduced retinal sensitivity in perimetry by about 2 dB [7].

Image forming and image analysis disorders in patients with diffractive intraocular lenses may potentially interfere with visual perception and associated cognitive processes. However, this study identified no statistically significant differences in the examined functions, which might suggest that the type of lens, multifocal or monocular, is not related to cognitive aspects of functioning. On the one hand, this outcome is probably attributable to the absence of such influence in patients well adapted to this type of lens. The average time elapsed since the operation in the study group was over one year, so the process of neuroadaptation had probably been completed. The results can be explained by good lighting conditions in which the cognitive tests were performed. It seems likely that visual disturbances would occur only in the case of low-intensity lighting. On the other hand, there are some limitations that might explain no statistically significant differences in cognitive function performance in patients with a different type of lenses. First of all, the sample size is insufficient to make any conclusion about the relation between cognitive function and type of lenses and differentiate this two group of patients based on the tests of psychomotor speed, attention, memory, control, and cognitive flexibility. Furthermore it is a very possible that the measurements of cognitive function, used in presented research, are too general. It would be advisable to use more specific test of visual skills, to examine more elementary aspects of perception like motor and visual perception skills and motor free visual perception skills.

\section{Conclusion}

The lack of statistical differences in TMT test and Stroop Test suggests that diffractive multifocal lens implantation does not significantly influence cognitive functions in comparison to monofocal intraocular lenses. Further studies in bigger samples with the use of more sophisticated methods are required to reveal potential subtle disturbances especially in poor light conditions.

\section{References}

1. Leyland M, Zinicola E. Multifocal versus monofocal intraocular lenses in cataract surgery: a systematic review. Ophthalmology. 2003; 110(9): 1789-1798, doi: 10.1016/S0161-6420(03)00722-X, indexed in Pubmed: 13129879

2. Madrid-Costa D, Cerviño A, Ferrer-Blasco T, et al. Visual and optical performance with hybrid multifocal intraocular lenses. Clin Exp Optom. 2010; 93(6): 426-440, doi: 10.1111/j.1444-0938.2010.00518.x, indexed in Pubmed: 20880314

3. Calladine D, Evans JR, Shah S, et al. Multifocal versus monofocal intraocular lenses after cataract extraction. Cochrane Database Syst Rev. 2012(9): CD003169, doi: 10.1002/14651858.CD003169.pub3, indexed in Pubmed: 22972061

4. de Silva SR, Evans JR, Kirthi V, et al. Multifocal versus monofocal intraocular lenses after cataract extraction. Cochrane Database Syst Rev. 2016; 12: CD003169, doi: 10.1002/14651858.CD003169.pub4, indexed in Pubmed: 27943250.

5. Alio JL, Plaza-Puche AB, Férnandez-Buenaga R, et al. Multifocal intraocular lenses: An overview. Surv Ophthalmol. 2017; 62(5): 611-634, doi: 10.1016/j.survophthal.2017.03.005, indexed in Pubmed: 28366683

6. Kamiya K, Hayashi K, Shimizu K, et al. Survey Working Group of the Japanese Society of Cataract and Refractive Surgery. Multifocal intraocular lens explantation: a case series of 50 eyes. Am J Ophthalmol. 2014; 158(2): 215-220.e1, doi: 10.1016/j.ajo.2014.04.010, indexed in Pubmed: 24792105.

7. Farid M, Chak G, Garg S, et al. Reduction in mean deviation values in automated perimetry in eyes with multifocal compared to monofocal intraocular lens implants. Am J Ophthalmol. 2014; 158(2): 227-231. e1, doi: 10.1016/j.ajo.2014.04.017, indexed in Pubmed: 24784872.

8. Nuzzi R, Tridico F. Comparison of visual outcomes, spectacles dependence and patient satisfaction of multifocal and accommodative intraocular lenses: innovative perspectives for maximal refractive-oriented cataract surgery. BMC Ophthalmol. 2017; 17(1): 12, doi: 10.1186/s12886-017-0411-9, indexed in Pubmed: 28196497.

9. Chen T, Yu F, Lin H, et al. Objective and subjective visual quality after implantation of all optic zone diffractive multifocal intraocular lenses: a prospective, case-control observational study. $\mathrm{Br} \mathrm{J}$ Ophthalmol. 2016; 100(11): 1530-1535, doi: 10.1136/bjophthalmol-2015-307135, indexed in Pubmed: 26903522

10. Peng C, Zhao J, Ma L, et al. Optical performance after bilateral implantation of apodized aspheric diffractive multifocal intraocular lenses with +3.00-D addition power. Acta Ophthalmol. 2012; 90(8): e586-e593, doi: 10.1111/j.1755-3768.2012.02497.x, indexed in Pubmed: 23194310

11. Ji J, Huang X, Fan X, et al. Visual performance of Acrysof ReSTOR compared with a monofocal intraocular lens following implantation in cataract surgery. Exp Ther Med. 2013; 5(1): 277-281, doi: 10.3892/etm.2012.740, indexed in Pubmed: 23251283.

12. Wilkins MR, Allan BD, Rubin GS, et al. Moorfields IOL Study Group. Randomized trial of multifocal intraocular lenses versus monovision after bilateral cataract surgery. Ophthalmology. 2013; 120(12): 2449-2455.e1, doi: 10.1016/j.ophtha.2013.07.048, indexed in Pubmed: 24070808

13. Pepin SM. Neuroadaptation of presbyopia-correcting intraocular lenses. Curr Opin Ophthalmol. 2008; 19(1): 10-12, doi: 10.1097/ICU .0b013e3282f31758, indexed in Pubmed: 18090890.

14. Lubiński W, Podboraczyńska-Jodko K, Gronkowska-Serafin J, et al. Visual outcomes three and six months after implantation of diffractive and refractive multifocal IOL combinations. Klin Oczna. 2011; 113(7-9): 209-215, indexed in Pubmed: 22256560.

15. Lezak, MD., Howieson DB., Loring DW. Neuropsychological Assessment. Oxford, Oxford University Press. ; 2004.

16. Hebb DA. Podręcznik Psychologii (Psychology). Warsaw, PWN. ; 1969.

17. Sánchez-Cubillo I, Periáñez JA, Adrover-Roig D, et al. Construct validity of the Trail Making Test: role of task-switching, working memory, inhibi- 
tion/interference control, and visuomotor abilities. J Int Neuropsycho Soc. 2009; 15(3): 438-450, doi: 10.1017/S1355617709090626, indexed in Pubmed: 19402930.

18. Strauss E, Sherman EMS, Spreen OA. compedium of neuropsychological tests: Administration, norms and commentator. Oxford, Oxford University Press, 2006:477-499. : 655-677.

19. Lamberty GJ, Putnam SH, Chatel DM, et al. Derived Trail Making Test indices: A preliminary report. Neuropsychiatry Neuropsychology and Behavioral Neurology. 1994; 7(3): 230-234
20. Rosa AM, Miranda ÂC, Patrício M, et al. Functional Magnetic Resonance Imaging to Assess the Neurobehavioral Impact of Dysphotopsia with Multifocal Intraocular Lenses. Ophthalmology. 2017; 124(9) 1280-1289, doi: 10.1016/j.ophtha.2017.03.033, indexed in Pubmed: 28433446

21. Palomino Bautista C, Carmona González D, Castillo Gómez A, et al Evolution of visual performance in 250 eyes implanted with the Tecnis ZM900 multifocal IOL. Eur J Ophthalmol. 2009; 19(5): 762-768, indexed in Pubmed: 19787595. 